\title{
Estimativas de expectativa de vida com doenças crônicas de coluna no Brasil
}

Estimates of life expectancy for individuals with chronic spinal diseases in Brazil

${ }^{1}$ Fundação João Pinheiro. Al. Acácias 70/sala 326C, São Luiz. 31.275-150

Belo Horizonte Minas

Gerais Brasil.

mirelacsc@gmail.com
Abstract Chronic spinal diseases, including deformities and muscular pain, are significant causes of morbidity among adults and the elderly. The scope of this study is to assess the life expectancy of Brazilians with chronic spinal diseases by sex and age between 2003 and 2008. The Sullivan method was used, combining the mortality/actuarial table with the prevalence of chronic spinal diseases. The mortality/actuarial tables published by the Brazilian Institute of Geography and Statistics (IBGE) were used and the prevalence of chronic spinal diseases was taken from the Brazilian Household Sample Survey (PNAD) for the years under scrutiny. The main results indicate that a man born in Brazil in 2008 could expect to live for 69.1 years, of which 15\% with chronic spinal diseases. However, women born in the same year had a life expectancy of 76.7 years and could expect to live a fifth of their lives with chronic spinal diseases. Over the period under analysis, concurrently with gains in life expectancy, there was an increase in healthy life expectancy, or length of life lived without chronic spinal diseases, both in absolute and relative terms.

Key words Healthy life expectancy, Chronic diseases, Spine, National Household Sample Survey (PNAD)
Resumo As doenças crônicas de coluna, entre elas as deformidades e as dores musculares, são importantes causas de morbidade entre adultos e idosos. O objetivo deste estudo foi estimar a expectativa de vida de brasileiros com doenças crônicas de coluna, por sexo e idade, nos anos de 2003 e 2008. Empregou-se o método de Sullivan, combinando a tábua de vida e as prevalências de doencas crônicas de coluna. Foram utilizadas as tábuas de vida publicadas pelo Instituto Brasileiro de Geografia e Estatística e as prevalências de enfermidades crônicas de coluna da Pesquisa Nacional por Amostra Domiciliar (PNAD), dos anos selecionados. Os principais resultados indicam que, no Brasil, ao nascer em 2008, um homem poderia esperar viver 69,1 anos, 15\% dos quais com doenças crônicas de coluna. Já as mulheres que nasceram neste mesmo ano, apresentavam uma expectativa de vida de 76,7 anos e esperariam viver um quinto de sua vida com problemas crônicos de coluna. No período analisado, concomitantemente aos ganhos na expectativa de vida, ocorreu um crescimento na expectativa de vida saudável, ou seja, nos anos de vida livre de doenças crônicas da coluna, tanto em termos absolutos como relativos.

Palavras-chave Expectativa de vida saudável, Doenças crônicas, Coluna, PNAD 


\section{Introdução}

Em 1940, a esperança de vida do brasileiro sequer atingia os 50 anos de idade $(45,5 \text { anos })^{1}$. Os avanços no setor da saúde e as melhorias nas condições gerais de vida da população concorreram para que, 71 anos mais tarde, este indicador atingisse 74,08 anos, em $2011^{2}$. Segundo a projeção de população do IBGE, em 2050 esse indicador deverá chegar aos 81,3 anos $^{1}$. Em uma época em que a população passou a viver mais, cresce $o$ interesse de investigar se o número adicional de anos acrescido à expectativa de vida é vivido em condições de saúde adequada.

Estimativas de expectativa de vida saudável têm sido utilizadas para suprir a demanda por informações sobre a quantidade de anos vividos com saúde. A esperança de vida saudável apresenta uma noção similar à expectativa de vida total, mas refere-se ao número médio de anos de vida que uma pessoa de determinada idade pode esperar viver com saúde, dado que prevaleçam as taxas de morbidade e mortalidade naquela idade específica. Sendo assim, a expectativa de vida total é composta pela quantidade de anos vividos, desde o nascimento ou a partir de uma determinada idade, em diferentes estados de saúde, até a morte, sendo que os anos vividos com saúde fornecem a expectativa de vida saudável ${ }^{3}$.

Assim como existem várias possibilidades para se definir saúde, há diferentes maneiras de se mensurar a expectativa de vida saudável como, por exemplo, a incapacidade funcional, a autopercepção de saúde e as doenças crônicas ${ }^{4}$. Sendo assim, a forma como o estado de saúde é operacionalizado depende dos objetivos do pesquisador e dos dados disponíveis. Diferentes formas de mensuração do estado saúde podem conduzir a resultados também distintos no que se refere à expectativa saudável.

No Brasil, recentemente, alguns estudos estimaram a expectativa de vida saudável ${ }^{5-12}$ empregando diferentes indicadores de saúde. Entretanto, ainda existe uma escassez de estudos brasileiros nessa área, que pode ser atribuída, em parte, à carência de informações, como aquela relativa às prevalências de doenças crônicas. Os suplementos de saúde da Pesquisa Nacional por Amostra de Domicílios (PNAD), realizada pelo IBGE, vieram suprir em parte a demanda por dados de prevalências de doenças crônicas, entre elas as de coluna. As condições crônicas mais prevalentes em 2008 foram: hipertensão, doença de coluna, artrite e depressão. A prevalência de ter ao menos uma doença crônica foi mais elevada em: idosos, mulheres, cor/raça preta ou indígena, menor escolaridade, migrantes, moradores em áreas urbanas e na região Sul do país ${ }^{13}$.

Em 2008, as doenças crônicas de coluna, identificadas por algum médico ou profissional de saúde, atingiam $13,5 \%$ da população brasileira e estavam no segundo lugar dentre as mais frequentemente declaradas, num total de 12 doenças crônicas investigadas pela $\mathrm{PNAD}^{14}$. Problemas crônicos de coluna, entre eles as deformidades e as dores musculares, são importantes causas de morbidade entre adultos e idosos, embora apresentem gravidade relativamente baixa. As alterações musculoesqueléticas ganharam maior relevância principalmente após a expansão da indústria, no século XIX, com a exacerbação de fatores de risco, como a postura estática, a inclinação constante do tronco para frente, o levantamento repetido de peso e o estresse ${ }^{15}$. Além disso, com o processo biológico de envelhecimento, desencadeia-se um processo natural de desgaste das estruturas da coluna, que dependendo do grau de comprometimento pode levar a alterações na mesma e dor ${ }^{16}$. Do ponto de vista pessoal, os problemas crônicos na coluna podem levar a perda do condicionamento físico, deterioração da saúde geral, diminuição da participação em atividades sociais, estresse familiar, diminuição do contato com a comunidade, perdas financeiras, irritação, ansiedade e depressão. Já em relação aos impactos econômicos, os gastos relacionados a essas patologias podem gerar prejuízos tanto para a sociedade como para o governo, como por exemplo, com o pagamento de tratamentos, de auxílio-doença e de aposentadoria por invalide $z^{17}$.

No caso específico do Brasil, a medida da expectativa de vida com doenças crônicas de coluna pode auxiliar no planejamento de políticas públicas, apontando algumas das necessidades reais da população e permitindo a alocação adequada de recursos humanos e financeiros. Afinal, esse indicador de saúde fornece informações não apenas sobre a prevalência, mas também sobre a duração potencial, mensurada pelos anos vividos com problemas crônicos de coluna, e o tempo necessário de tratamento e cuidados por parte da população $0^{6,18}$.

Para estimar a esperança de vida com problemas de coluna ao nascer, por exemplo, são empregados não apenas as prevalências de problemas de coluna e riscos de morte na primeira idade, mas para as fases de infância, adolescência, juventude, idade adulta e velhice. Assim, com esta medida síntese é possível estimar quantos anos, em média, uma pessoa viverá com proble- 


\section{Metodologia}

\section{Fonte de Dados}

Este estudo utiliza dados da Pesquisa Nacional por Amostra de Domicílios (PNAD) dos anos de $2003^{19}$ e $2008^{20}$ e Tábuas de Vida Completas, por sexo, publicadas pelo IBGE para os mesmos $\operatorname{anos}^{21,22}$.

Implantada em 1967, a PNAD investiga anualmente, de forma permanente, características gerais da população, de educação, trabalho, rendimento e habitação. Em 2003 e 2008, anos considerados neste estudo, a PNAD teve um suplemento especial de saúde, que dentre outras informações, permitiu obter os dados sobre doenças crônicas de coluna ${ }^{19,20}$.

As informações contidas no suplemento de saúde da PNAD são amplamente utilizadas, proporcionando o conhecimento sobre as características de saúde da população brasileira em diversos aspectos como: acesso e utilização de serviços de saúde, realização de exames preventivos da saúde por mulheres, realização de cirurgia para retirada do útero, mobilidade física, doenças crônicas, fatores de risco e proteção da saúde da população (atividade física, tabagismo, trânsito e violência), entre outros ${ }^{13,23}$. No questionário foram incluídas perguntas sobre 12 doenças crônicas: 1) doença de coluna ou costas; 2) artrite ou reumatismo; 3) câncer; 4) diabetes; 5) bronquite ou asma; 6) hipertensão (pressão alta); 7) doença do coração; 8) doença renal crônica; 9) depressão; 10) tuberculose; 11) tendinite ou tenossinovite; e 12) cirrose.

Na PNAD, doença de coluna ou costas é definida por problema crônico nestes locais por enfermidade, desvio, curvatura anormal (escoliose, cifose, lordose) ou deformidade na coluna vertebral (cervical, dorsal, lombar), como, por exemplo, artrose ou osteoporose localizada na coluna, hérnia de disco, bico de papagaio, etc., inclusive dor nas costas causada por esforço muscular. Salienta-se que, assim como para as demais doenças crônicas, cabia ao entrevistador esclarecer que o problema deve acompanhar a pessoa por um longo período de tempo, poden- do ter fases agudas, momentos de piora ou melhora sensível, o que dá ao entrevistado a noção de que se trata de uma doença crônica ${ }^{19,20}$.

Neste estudo, foram utilizadas prevalências de doenças crônicas de coluna, por sexo e grupo etário, contidas no módulo especial de saúde da PNAD. A utilização dos dados de 2003 e 2008 é interessante, pois permite a análise em dois pontos do tempo, com intervalo de cinco anos.

Inicialmente, pensou-se em incluir na análise o ano de 1998. Entretanto, houve uma alteração na forma de perguntar sobre problema crônico de coluna. Em 1998, foi perguntado se o sujeito tem doença de coluna ou costas? Já em 2003 e 2008 se algum médico ou profissional de saúde disse que o indivíduo tem doença de coluna ou costas? A variação na forma de perguntar certamente não é desejável, uma vez que pode gerar alteração na forma em que o sujeito responde a pergunta. Afinal, supostamente, na segunda pergunta o entrevistado responde sobre um diagnóstico que lhe foi fornecido e no primeiro não há esta necessidade. Procedeu-se então com a análise das prevalências para decidir sobre a utilização desses dados. Como não há como ter certeza se a redução das prevalências pode ser atribuída à melhora nas condições de saúde da população ou à alteração no modo de perguntar, decidiu-se por não manter as estimativas de expectativa de vida saudável para o ano de 1998, uma vez que não seriam feitas comparações.

Foram classificados como presença de doença crônica de coluna, aqueles indivíduos que responderam sim à pergunta destinada a esta doença crônica. Em caso negativo ou de pessoas que não souberam ou não responderam foram qualificados como ausência de problemas crônicos de coluna. Para compor a amostra, foram incluídas pessoas de todas as faixas etárias, excluindo os casos sem declaração de idade. A amostra empregada neste estudo foi composta por: 384.762 pessoas em 2003 (187.542 homens e 197.220 mulheres $)^{19}$ e 391.868 pessoas em 2008 $\left(190.580\right.$ homens e 201.288 mulheres ${ }^{20}$. Para calcular as prevalências, foram utilizados os pesos existentes na própria base, para garantir a representatividade da população total.

Finalmente, as outras fontes de informações empregadas são as Tábuas de Vida Completas publicadas pelo IBGE ${ }^{21,22}$. Essas são construídas anualmente pelo instituto e são uma projeção com base na mortalidade calculada para os anos do Censo Demográfico, as quais resultaram de uma ampla discussão entre seus técnicos. 


\section{Método}

Para estimar a expectativa de vida com doenças crônicas de coluna foi utilizado o método elaborado por Sullivan ${ }^{24}$, combinando a tábua de vida com experiência de mortalidade corrente da população em 2003 e 2008, e as prevalências de doenças crônicas de coluna na população no mesmo período.

A expectativa de vida com doenças crônicas de coluna $\left(\mathrm{EVCDC}_{\mathrm{x}}\right)$ é definida como:

$$
E V C P C_{x}=\frac{\sum\left({ }_{n} \pi_{x}\right)_{n} L_{x}}{l_{x}}
$$

Onde:

EVCDC : Expectativa de vida com doenças crônicas de coluna, que corresponde ao número médio de anos a serem vividos com problemas crônicos de coluna a partir da idade $\mathrm{x}$;

${ }_{n} \pi_{x}$ : proporção de pessoas com doenças crônicas de coluna no grupo etário $\mathrm{x} \mathrm{a} \mathrm{x}+\mathrm{n}$;

${ }_{n} L_{x}$ : pessoas-anos vividos de $\mathrm{x}$ a $\mathrm{x}+\mathrm{n}$, que corresponde ao total de anos vividos pela coorte no intervalo;

$1_{\mathrm{x}}$ : probabilidade de sobreviver até a idade $\mathrm{x}$.

Já a expectativa de vida livre de doenças crônicas de coluna (EVLDC $C_{x}$, que fornece o número médio de anos a serem vividos livres de problemas crônicos de coluna a partir de determinada idade, foi obtida diretamente subtraindo da expectativa de vida total $\left(\mathrm{EV}_{\mathrm{x}}\right)$ a EVCDC : $E V L P C_{x}=E V_{x}-$ EVCPC . Outra opção seria empregar o Método de Sullivan ${ }^{24}$ e, neste caso, utilizar a proporção de pessoas livres de doenças crônicas de coluna no grupo etário $x$ a $x+n$. Além disso, estimou-se a proporção de anos a serem vividos livres de doenças crônicas de coluna (\% EVLDC ) e com doenças crônicas de coluna (\% EVCDC $_{\gamma}$ ), pela razão entre o número de anos a serem vividos em cada uma dessas condições e o total de anos a serem vividos.

As tabelas de sobrevivência foram construídas separadamente para cada ano e sexo, sendo o número de anos vividos dentro de cada idade na tábua de vida distribuído segundo a prevalência de doenças crônicas de coluna em cada grupo etário específico. A opção por estimar as prevalências por grupos quinquenais teve como objetivo minimizar possíveis erros de estimativas por idade simples. Para apresentação dos resultados foram selecionadas as estimativas ao nascer, 20, 40 e 60 anos.

\section{Resultados}

Na Tabela 1, são apresentadas as prevalências dos problemas crônicos de coluna na população brasileira, de acordo com características sociodemográficas e de saúde, em 2003 e 2008. Embora este artigo não se proponha analisar fatores associados a esta enfermidade, tampouco relações existentes entre as diversas variáveis, julgouse oportuno contextualizar os problemas crônicos de coluna por meio das prevalências.

No Gráfico 1 são apresentadas as prevalências de doenças crônicas de coluna por grupo etário para os anos de 2003 e 2008. Independente do ano analisado e principalmente em idades mais avançadas, o gráfico chama a atenção para as diferenças entre os sexos nas prevalências de doenças crônicas de coluna, sendo este tipo de enfermidade mais prevalente entre as mulheres. Quando analisados os anos de 2003 e 2008, para ambos os sexos, nota-se uma ligeira melhora nas prevalências em 5 anos.

Nas Tabelas 2 e 3 apresenta-se uma síntese das estimativas para o Brasil de expectativa de

Tabela 1. Prevalência de problemas crônicos de coluna segundo características sociodemográficas e de saúde, Brasil 2003 e 2008 - (\%).

\begin{tabular}{lcc}
\hline & $\mathbf{2 0 0 3}$ & $\mathbf{2 0 0 8}$ \\
\hline Sexo & & \\
$\quad$ Homens & 11,1 & 11,4 \\
$\quad$ Mulheres & 15,4 & 15,4 \\
Cor ou raça & & \\
$\quad$ Branca & 14,1 & 14,1 \\
Preta & 14,1 & 14,5 \\
Parda & 12,1 & 12,5 \\
Escolaridade (anos de estudo) & & \\
Sem instrução e menos de 1 ano & 11,0 & 11,8 \\
1 a 3 anos & 14,1 & 15,1 \\
4 a 7 anos & 14,5 & 15,2 \\
8 a 10 anos & 12,2 & 12,4 \\
11 a 14 anos & 13,6 & 12,6 \\
15 anos ou mais & 17,3 & 14,6 \\
Região & & \\
Norte & 10,8 & 11,2 \\
Nordeste & 12,3 & 12,6 \\
Sudeste & 13,5 & 13,8 \\
Sul & 15,1 & 15,1 \\
Centro-Oeste & 14,0 & 13,8 \\
Autopercepção da saúde & & \\
Muito boa/boa & 48,2 & 43,7 \\
Regular & 40,8 & 43,3 \\
Ruim/Muito ruim & 11,0 & 13,0 \\
\hline & & \\
\hline & &
\end{tabular}

Fonte: Instituto Brasileiro de Geografia e Estatística ${ }^{19,20}$. 


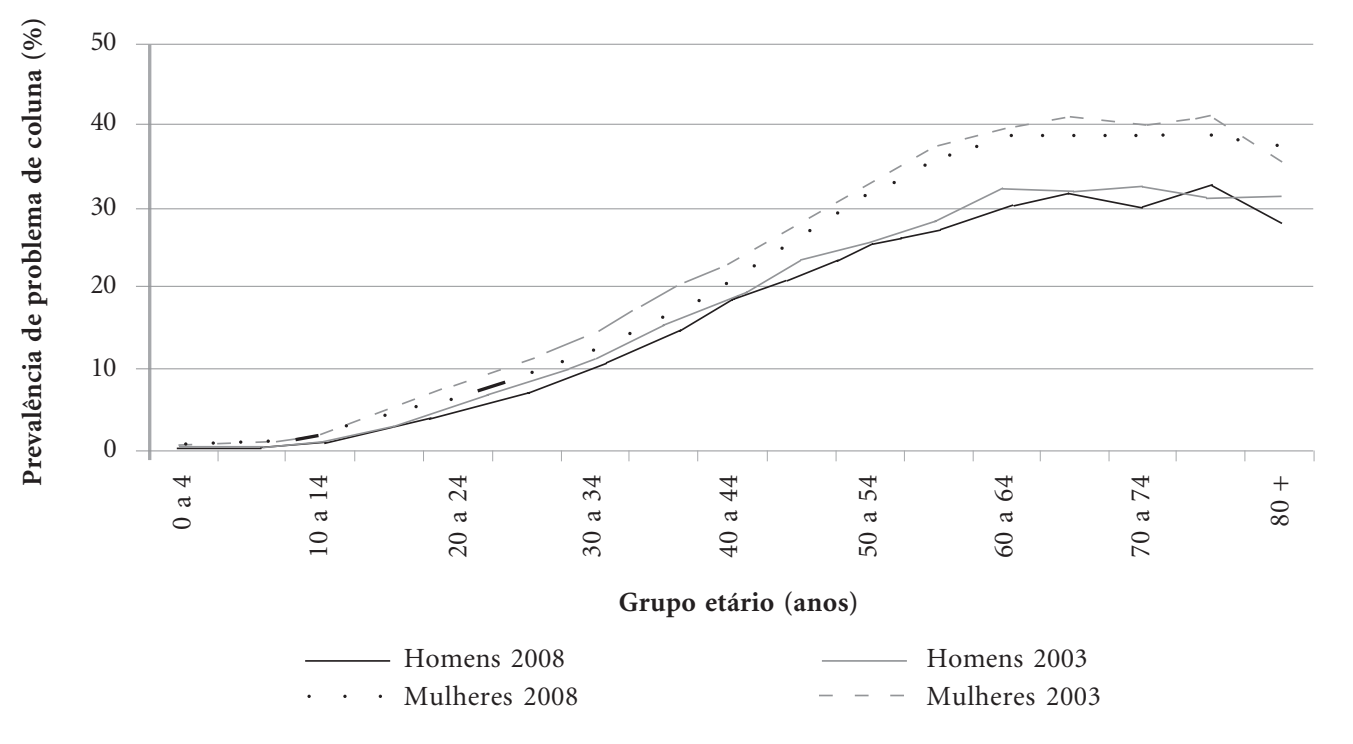

Gráfico 1. Prevalências de doenças crônicas de coluna por grupos etários, Brasil, 2003 e 2008 - (\%).

Fonte: Instituto Brasileiro de Geografia e Estatística ${ }^{19,20}$.

Tabela 2. Estimativas de expectativa de vida total $\left(\mathrm{EV}_{\mathrm{x}}\right)$, livre de doenças crônicas de coluna ( $\left.\mathrm{EVLDC}_{\mathrm{x}}\right)$ e com doenças crônicas de coluna $\left(\mathrm{EVCDC}_{\mathrm{x}}\right.$ ) para mulheres ao nascer, 20, 40 e 60 anos, Brasil, 2003 e 2008.

\begin{tabular}{ccccccc}
\hline Ano & Idade $(\mathbf{x})$ & $\mathbf{E V}_{\mathbf{x}}$ & EVLDC $_{\mathbf{x}}$ & EVCDC $_{\mathbf{x}}$ & \% EVLDC $_{\mathbf{x}}$ & \% EVCDC $_{\mathbf{x}}$ \\
\hline 2008 & 0 & 76,7 & 61,4 & 15,3 & 80 & 20 \\
& 20 & 58,9 & 43,5 & 15,4 & 74 & 26 \\
& 40 & 39,8 & 26,4 & 13,5 & 66 & 34 \\
2003 & 60 & 22,7 & 13,9 & 8,7 & 61 & 39 \\
& 0 & 75,2 & 59,5 & 15,7 & 79 & 21 \\
& 20 & 57,8 & 41,9 & 15,9 & 73 & 27 \\
& 40 & 39,0 & 25,4 & 13,6 & 65 & 35 \\
& 60 & 22,1 & 13,4 & 8,7 & 61 & 39 \\
\hline
\end{tabular}

Fonte: Instituto Brasileiro de Geografia e Estatística ${ }^{19-22}$.

Tabela 3. Estimativas de expectativa de vida total $\left(\mathrm{EV}_{\mathrm{x}}\right)$, livre de doenças crônicas de coluna $\left(\mathrm{EVLDC}_{\mathrm{x}}\right) \mathrm{e}$ com doenças crônicas de coluna $\left(\mathrm{EVCDC}_{\mathrm{x}}\right.$ ) para homens ao nascer, 20, 40 e 60 anos, Brasil, 2003 e 2008.

\begin{tabular}{ccccccc}
\hline Ano & Idade $(\mathbf{x})$ & $\mathbf{E V}_{\mathbf{x}}$ & EVLDC $_{\mathbf{x}}$ & EVCDC $_{\mathbf{x}}$ & \% EVLDC $_{\mathbf{x}}$ & \% EVCDC $_{\mathbf{x}}$ \\
\hline 2008 & 0 & 69,1 & 59,0 & 10,1 & 85 & 15 \\
& 20 & 52,1 & 41,7 & 10,4 & 80 & 20 \\
& 40 & 34,8 & 25,6 & 9,2 & 74 & 26 \\
2003 & 60 & 19,5 & 13,6 & 5,9 & 70 & 30 \\
& 0 & 67,6 & 57,3 & 10,3 & 85 & 15 \\
& 20 & 51,0 & 40,4 & 10,7 & 79 & 21 \\
& 40 & 34,1 & 24,7 & 9,4 & 73 & 32 \\
\hline
\end{tabular}

Fonte: Instituto Brasileiro de Geografia e Estatística ${ }^{19-22}$. 
vida total, livre e com doenças crônicas de coluna para diversas idades selecionadas (ao nascer, 20, 40 e 60 anos) em 2003 e 2008, para mulheres e homens, respectivamente.

Em 2008, ao nascer, uma mulher poderia esperar viver em média 76,7 anos, dos quais $20 \%$ (15,3 anos) seriam vividos com doenças crônicas de coluna. Neste mesmo ano, ao atingir 60 anos, a proporção de anos vividos nesta condição dobraria, sendo que 8,7 anos, dos 22,7 anos que poderia esperar viver, seriam com problemas crônicos de coluna.

Analisando os dois anos selecionados, observa-se que, para as mulheres, no quinquênio 2003 a 2008, houve um aumento de 1,5 ano na esperança de vida ao nascer. Pensando no que significaria este aumento em termos de condições de saúde, pode-se constatar que representou um crescimento de anos de vida saudável para a população feminina. Afinal, observa-se um aumento nos anos a serem vividos livre de problemas crônicos de coluna. De 2003 para 2008, a redução de 0,4 anos a serem vividos com doenças crônicas de coluna somada ao aumento da esperança de vida, resultou em um aumento de 1,9 ano na expectativa de vida ao nascer livre de doenças crônicas de coluna.

Em relação aos homens, que apresentam expectativas de vida menores que as mulheres em todas as idades observadas e anos analisados, nota-se uma proporção menor de anos vividos com doenças crônicas de coluna. Em 2008, por exemplo, a esperança de vida ao nascer era de 69,1 anos e a expectativa de vida com doenças crônicas de coluna atingiu $15 \%$ dos anos a serem vividos (10,1 anos). Assim, observa-se que as mulheres vivem mais, porém passam um maior tempo com problemas crônicos de coluna, o que é verdade tanto em termos absolutos como relativos.

Em termos de ganhos de anos de vida saudável, observa-se que estes foram praticamente iguais entre os sexos em termos proporcionais. No quinquênio 2003 a 2008, em números absolutos, a redução dos anos a serem vividos com problemas crônicos de coluna ao nascer foi menor para os homens, embora os ganhos na esperança de vida total tenham sido iguais.

Finalmente, nos anos analisados, para homens e mulheres, o tempo a ser vivido livre de doenças crônicas de coluna, ou seja, os anos de vida saudáveis, foi sempre maior que os anos a serem vividos com problemas crônicos de coluna.

\section{Discussão}

Este artigo estimou os anos a serem vividos com e sem doenças crônicas de coluna para diversas idades. Em média, as mulheres apresentam maiores expectativas de vida. Entretanto, se comparadas aos homens, elas possuem uma menor proporção de anos a serem vividos sem doenças crônicas de coluna, independentemente do período analisado. Deste modo, se por um lado, em relação aos homens, as mulheres vivem mais, por outro, elas passam um número maior de anos com deformidades e dores musculares relacionadas a doenças crônicas na coluna, o que se observa em todas as idades analisadas, em termos relativos e absolutos. Os resultados apresentados mostraram que, com o avançar da idade, as diferenças entre os sexos diminuem no caso da expectativa de vida com doenças crônicas de coluna, embora aumentem quando analisadas em termos proporcionais. Mesmo que a forma de mensurar saúde possa variar entre os estudos, dificultando comparações, os resultados do presente estudo corroboram trabalhos prévios que destacaram a desvantagem feminina em relação à expectativa de vida saudável ${ }^{5-10,16,25}$.

A respeito da discussão entre as diferenças entre os sexos em relação à expectativa de vida saudável, o que os estudos têm sugerido é que taxas de mortalidade mais elevadas dos homens em idades mais jovens poderiam permitir que, na velhice, a composição do grupo etário masculino fosse mais favorável que o feminino, devido a um mecanismo de seleção ${ }^{26}$. Isto resultaria num grupo heterogêneo no qual haveria um número maior de idosas mais fragilizadas e susceptíveis do que idosos, o que seria refletido diretamente nos anos a serem vividos com saúde debi$\operatorname{litada}^{6-10}$. Ainda sobre as diferenças entre homens e mulheres, acredita-se que um dos principais fatores explicativos seria a maior sobrevivência feminina, permitindo que elas atinjam idades mais avançadas, nas quais o surgimento de doenças crônicas e suas consequências são mais evidentes. Além disso, sugere-se que as condições de saúde das mulheres podem ser reflexo de condições econômicas, sociais e culturais desiguais, nos diversos momentos de suas vidas ${ }^{27,28}$. Historicamente, as mulheres ainda são as principais responsáveis pelas tarefas domésticas e cuidados com crianças. Diante do aumento da participação feminina no mercado de trabalho, configura 
a necessidade de se conciliar as atividades produtivas e as reprodutivas (relativas à família). Além disso, persistem as diferenças de rendimentos entre homens e mulheres, oriundas, entre outros fatores, das distinções entre os gêneros na distribuição entre as diferentes ocupações, dotadas de status desiguais ${ }^{29}$.

Os resultados mostraram que ocorreram concomitantemente aos ganhos na expectativa de vida, um crescimento na expectativa de vida livre de doenças crônicas da coluna, tanto em termos absolutos como relativos. Ou seja, além de viver mais, a população brasileira poderia esperar viver um número maior de anos com saúde. Para homens e mulheres, os ganhos no tempo vivido com saúde, ou livre de doenças crônicas de coluna, foram maiores que os obtidos para a esperança de vida ao nascer, 20, 40 e 60 anos.

Contudo, algumas limitações deste trabalho devem ser ressaltadas. Em primeiro lugar, por não empregar dados longitudinais, não foram incorporadas às estimativas possíveis mudanças em relação a melhorias nas condições de saúde e nas taxas de mortalidade da população nos diferentes anos analisados. Este, porém, não é um problema inerente ao método, e sim à construção das tábuas de vida de período. Deve-se ressaltar que o método de Sullivan permite comparar a saúde entre diferentes populações e também de uma mesma em períodos distintos, além disso, estudos prévios têm demonstrado que, caso não existam alterações repentinas, tanto nas prevalências como nas taxas de mortalidade, seja bastante confiável para este tipo de análise ${ }^{30}$. Sendo assim, acredita-se que as estimativas realizadas aqui podem ser utilizadas para representar a realidade da população brasileira.

Outra limitação do estudo é que, assim como para as demais questões referentes à saúde, em apenas $38,2 \%$ dos casos em 2003 e em $48,8 \%$ em 2008, o informante da pergunta sobre a presença de doença crônica de coluna era a própria pessoa. Contudo, em apenas $2,1 \%$ dos casos em 2003 e em $1,9 \%$ em 2008 , era uma pessoa que não morava no domicílio que respondia a questão.

Finalmente, pode-se considerar como limitação a utilização de dados de uma pesquisa que não foi desenhada especificamente para acompanhar doenças crônicas na coluna. Este fato deixa o conceito de doença crônica de coluna amplo, aplicável tanto a deformidades como a dores musculares. Entretanto, é importante ressaltar que trata-se de uma primeira tentativa de analisar o número de anos a serem vividos nessas condições e que, infelizmente, os dados exis- tentes para o Brasil não permitem estimar essas prevalências separadamente, analisando, por exemplo, os anos a serem vividos com dores lombares, uma importante causa de morbidade. Mesmo considerando suas limitações, ressaltase a inovação ao utilizar na construção das estimativas os dados da PNAD, uma pesquisa com representatividade nacional. Ademais, foi possível fazer comparações em dois pontos do tempo, uma vez que a pesquisa utiliza uma amostra probabilística, com o mesmo plano de amostragem e as mesmas perguntas.

Mesmo que o tempo a ser vivido com problemas crônicos de coluna seja menor que o vivido livre dessas condições é preciso pensar na demanda de cuidado requerida por esta população. Afinal, em média, considerando a expectativa de vida ao nascer em 2008, uma mulher brasileira vai demandar de 15 anos e um homem de 10 anos de acompanhamento clínico constante, o que refletirá diretamente nos gastos com cuidado e reabilitação. As estimativas realizadas permitem refletir sobre os anos a serem vividos com necessidade de: consultas médicas com ortopedistas; tratamento ambulatorial com fisioterapeutas; medicamentos e exames diversos; afastamentos da atividade laboral; cuidados dentro da família; entre outros. Além de todas essas demandas, destaca-se a perda na qualidade de vida dos indivíduos.

Este tipo de discussão reforça a ideia de que, tanto para a população como para o Estado, os investimentos em prevenção que sejam capazes de reduzir o tempo vivido com problemas crônicos de coluna, como as deformidades e as dores musculares, são ainda a melhor solução para reduzir custos e ampliar a qualidade de vida nos anos remanescentes.

\section{Conclusões}

Este artigo apresenta as primeiras estimativas de expectativa de vida com e sem doenças crônicas de colunas, para brasileiros de ambos os sexos. Os resultados mostraram que, no Brasil, ao nascer em 2008, um homem poderá esperar viver 69,1 anos, $15 \%$ dos quais com doenças crônicas de coluna. Já as mulheres que nasceram neste mesmo ano, apresentavam uma expectativa de vida de 76,7 anos e esperariam viver um quinto de sua vida com problemas crônicos de coluna. No período analisado, concomitantemente aos ganhos na expectativa de vida, ocorreu um crescimento na de vida saudável, ou seja, nos anos de 
vida livre de doenças crônicas da coluna, tanto em termos absolutos como relativos.

$\mathrm{Na}$ medida em que apresenta informações sobre os anos a serem vividos com e sem doenças crônicas de coluna, o presente estudo fornece subsídios para se estimar a demanda por cuidado e intervenções de saúde da população, apontando para a necessidade de se investir em prevenção para que a expectativa de vida livre de problemas crônicos de coluna possa ser ampliada. Além disso, reforça a necessidade de considerar as diferenças entre os sexos em relação à demanda por cuidados de saúde.

\section{Agradecimentos}

A autora agradece a Fundação de Amparo a Pesquisa do Estado de Minas Gerais (FAPEMIG) pelo apoio para publicação deste artigo.

\section{Referências}

1. Instituto Brasileiro de Geografia e Estatística (IBGE). Em 2008, esperança de vida dos brasileiros chega a 72,86 anos. 2009. [acessado 2011 maio 5]. Disponível em: http://www.ibge.gov.br/home/presidencia/ noticias/noticia_visualiza.php?id_noticia $=1507$ \&id_pagina $=1$

2. Instituto Brasileiro de Geografia e Estatística (IBGE). Tábuas Completas de Mortalidade - 2011. [acessado 2013 maio 13]. Disponível em: http://www.ibge.gov. br/home/estatistica/populacao/tabuadevida/2011/ default.shtm

3. Jagger C. Health expectancy calculation by the Sullivan Method: a practical guide. Madison: NUPRI; 1999. (NUPRI Research Paper, no 68)

4. Robine J-M, Romieu I, Cambois E. Health expectancy indicators. Bull World Health Organ 1999; 77(3):181-185.

5. Baptista DBDA. Idosos no município de São Paulo: expectativa de vida ativa e perfis multidimensionais de incapacidade a partir da SABE [dissertação]. Belo Horizonte: Universidade Federal de Minas Gerais; 2003.

6. Camargos MCS, Perpétuo IHO, Machado CJ. Expectativa de vida com incapacidade funcional em idosos em São Paulo, Brasil. Rev Panam Salud Publica 2005; 17(5-6):379-386.

7. Camargos MCS, Machado CJ, Rodrigues, RN. Disability life expectancy for the elderly, city of São Paulo, Brazil, 2000: gender and educational differences. J Biosoc Sci 2007; 39(3):455-463.

8. Camargos MCS, Machado CJ, Rodrigues, RN. Life expectancy among elderly Brazilians in 2003 according to different levels of functional disability. Cad Saude Publica 2008; 24(4):845-852.

9. Camargos MCS, Machado CJ, Rodrigues, RN. Sex differences in healthy life expectancy from self-perceived assessments of health in the City of São Paulo, Brazil. Ageing Soc 2008; 28(1):35-48. 
10. Camargos MCS, Rodrigues, RN, Machado CJ. Expectativa de vida saudável para idosos brasileiros, 2003. Cien Saude Colet 2009; 14(5):1903-1909.

11. Romero DE, Leite IC, Szwarcwald CL. Healthy life expectancy in Brazil: applying the Sullivan method. Cad Saude Publica 2005; 21(Supl. 1):S7-S18.

12. Andrade FCD, Guevara PE, Lebrão, ML, Duarte YAO, Santos JLF. Gender Differences in Life Expectancy and Disability-Free Life Expectancy Among Older Adults in São Paulo, Brazil. Women's Health Issues 2011; 21(1):64-70.

13. Barros MBA, Francisco PMSB, Zanchetta, LM, César, CLG. Tendências das desigualdades sociais e demográficas na prevalência de doenças crônicas no Brasil, PNAD: 2003- 2008. Cien Saude Colet 2011; 16(9):3755-3768.

14. Instituto Brasileiro de Geografia e Estatística (IBGE). Um Panorama da Saúde no Brasil - Acesso e utilização dos serviços, condições de saúde e fatores de risco e proteção à saúde 2008. 2010. [acessado 2011 maio 5]. Disponível em: http://www.ibge.gov.br/home/ estatistica/populacao/panorama_saude_brasil 2003_2008/PNAD_2008_saude.pdf

15. Pontes, H. A Incidência da Lombalgia em Indústria de Fundicão: um estudo de caso sob a ótica da ergonomia [dissertação]. Ponta Grossa: Universidade Tecnológica Federal do Paraná; 2005.

16. Silva MC, Fassa AG, Valle NCJ. Dor lombar crônica em uma população adulta do Sul do Brasil: prevalência e fatores associados. Cad Saude Publica 2004; 20(2):377-385.

17. Ferreira MS, Navega MT. Efeitos de um programa de orientação para adultos com lombalgia. Acta Ortop Bras 2010; 18(3):127-131.

18. Agree EM. The influence of personal care and assistive devices on the measurement of disability. Soc Sci Med 1999; 48(4):427-443.

19. Instituto Brasileiro de Geografia e Estatística (IBGE) Pesquisa Nacional por Amostra de Domicílios, 2003. Rio de Janeiro: IBGE; 2003.

20. Instituto Brasileiro de Geografia e Estatística (IBGE). Pesquisa Nacional por Amostra de Domicílios, 2008. Rio de Janeiro: IBGE; 2008.

21. Instituto Brasileiro de Geografia e Estatística (IBGE). Tábuas Completas de Mortalidade - 2003. [acessado 2010 nov 10] Disponível em: ftp://ftp.ibge.gov.br/ Tabuas_Completas_de_Mortalidade/Tabuas_ Completas_de_Mortalidade_2003/
22. Instituto Brasileiro de Geografia e Estatística (IBGE) Tábuas Completas de Mortalidade - 2008. [acessado 2011 mar 11]. Disponível em: http://www.ibge. gov.br/home/estatistica/populacao/tabuadevida/ 2008/default.shtm

23. Viacava F, Dachs N, Travassos C. Os inquéritos domiciliares e o Sistema Nacional de Informações em Saúde. Cien Saude Colet 2006; 11(4):863-869.

24. Sullivan DF. A single index of mortality and morbidity. HSMHA Health Reports 1971; 86(4):347-354.

25. Zimmer Z. Active life expectancy and functional limitations among older Cambodians: results from a 2004 survey. New York: Population Council; 2005. (Working Papers No. 201).

26. Perls T, Kunkel LM, Puca, AA. The genetics of exceptional human longevity. J Mol Neurosci 2002; 19(1-2):233-238.

27. Barreto SM, Giatti L, Uchôa E, Lima-Costa, MF. Gênero e desigualdades em saúde entre idosos brasileiros. In: Anais da Oficina de Trabalho sobre Desigualdades Sociais e de Gênero em Saúde de Idosos no Brasil. Ouro Preto: Ministério da Saúde, Fundacão Oswaldo Cruz, Universidade Federal de Minas Gerais; 2002. p. 59-69.

28. Goldani AM. Mulheres e envelhecimento: desafios para novos contratos intergeracionais e de gênero. In: Camarano AA, organizadora. Muito além dos 60: os novos idosos brasileiros. Rio de Janeiro: IPEA; 1999. p. 75-113.

29. Fundação João Pinheiro. Trabalho e gênero. Boletim PAD - MG/2011. Trabalho e gênero. Belo Horizonte: Fundação João Pinheiro; 2013. 1(5).

30. Mathers CD, Robine JM. How good is Sullivan's method for monitoring changes in population health expectancies? J Epidemiol Community Health 1997; 51(1):80-86.

Artigo apresentado em 10/07/2013

Aprovado em 27/08/2013

Versão final apresentada em 02/09/2013 